Rapid Reviews COVID-19

\title{
Review 1: "A Sew-Free Origami Mask for Improvised Respiratory Protection"
}

\section{Cary Hill ${ }^{1}$}

${ }^{1}$ Vice President, NanoSafe, Inc., USA

Published on: Oct 30, 2020

DOI: $10.1162 / 2 \mathrm{e} 3983 f 5.91 \mathrm{e} 80 \mathrm{~cd} 2$

License: Creative Commons Attribution 4.0 International License (CC-BY 4.0). 


\section{$\underline{\text { RR:C19 Evidence Scale rating by reviewer: }}$}

- Strong. The main study claims are very well-justified by the data and analytic methods used. There is little room for doubt that the study produced has very similar results and conclusions as compared with the hypothetical ideal study. The study's main claims should be considered conclusive and actionable without reservation.

$* * * * * * * * * * * * * * * * * * * * * * * * * * * * * * * * * * * * * * * *$

\section{Review:}

The authors present an innovative and accessible means for the construction of masks for respiratory protection in the context of limited commercial supply, as experienced during the COVID-19 pandemic. The process for making the sew-free origami mask is clearly described in a manner that would be easily replicable by a broad readership.

Further considerations corresponding to fit and comfort provide additional appeal; these include the increase in dead space volume between face and mask over common flat mask designs (those similar to surgical masks) and the use of head-anchored straps rather than ear loops. Increased dead space is suggested to reduce $\mathrm{CO}_{2}$ accumulation rate from respired breath and reduce shifting of the mask during oral activities such as speaking. Head-anchored straps enable a tighter overall fit compared with ear loops, which can be an important factor in reducing leakage.

The methods used to test the presented mask design are robust and commensurate with current research ${ }^{1-5}$. The testing apparatus is clearly illustrated, and materials and equipment used for aerosol generation and characterization are common and proven in this field of study. A significant number of filter materials are tested including those from commercially available masks for comparison, providing satisfactory context for conclusions. Data is presented clearly and accessibly; produced results agree with and improve upon the understanding presented by existing research. A minor critique is that the characterization of the baseline (background) aerosol is not provided beyond mention that $\mathrm{NaCl}$ particles in the 50-700 diameter range were targeted. A concentration versus diameter curve for the background aerosol would be instructive to allow for enhanced comparison between published studies.

The conclusions drawn by the authors are reasonable and informative. It is of specific value that the impact of air permeability of filter media on ultimate mask filtration performance is emphasized and supported by acquired data. The data and conclusions 
presented in this work agree strongly with concurrent independent work conducted and recently published by this reviewer 6 . The discovery that materials exhibiting lower ideal filtration efficiency but higher permeability can actually provide greater overall filtration efficiency (and therefore aerosol protection) when in mask form than high filtration/low permeability materials is crucial; established mask testing standards prescribe testing of masks in ideal, sealed scenarios only 7,8 , which can be misleading since common masks (and even many N95 respirators) do not fit tightly enough to create a perfect seal. Namely, the authors demonstrate certain materials to exhibit an ideal filtration efficiency of $\sim 80 \%$, with a filtration efficiency of $\sim 65 \%$ in mask form. This is actually much higher than the surgical and KN95 masks, which exhibit $>90 \%$ ideal filtration efficiency but closer to $30 \%$ filtration efficiency in mask form.

Overall, the reported findings are of high impact in the field of improvised personal protective equipment, with implications that reach into the realm of commercial personal protective equipment. Data is collected competently, results are presented clearly, and conclusions are drawn reasonably. These results should inform readers to carefully select materials that are permeable enough to allow airflow and designs that minimize leakage; the presented origami mask is demonstrated to be an excellent candidate for such purposes.

1 Davies, A. et al. Testing the efficacy of homemade masks: would they protect in an influenza pandemic? Disaster Med Public Health Prep 7, 413-418, doi:10.1017/dmp.2013.43 (2013).

2 Konda, A. et al. Aerosol Filtration Efficiency of Common Fabrics Used in Respiratory Cloth Masks. ACS Nano, doi:10.1021/acsnano.0c03252 (2020).

3 Weber, A. et al. Aerosol penetration and leakage characteristics of masks used in the health care industry. American journal of infection control 21, 167-173, doi:10.1016/0196-6553(93)90027-2 (1993).

4 Zangmeister, C. D., Radney, J. G., Vicenzi, E. P. \& Weaver, J. L. Filtration Efficiencies of Nanoscale Aerosol by Cloth Mask Materials Used to Slow the Spread of SARS-CoV-2. ACS Nano, doi:10.1021/acsnano.0c05025 (2020).

5 Rengasamy, S., Eimer, B. \& Shaffer, R. E. Simple Respiratory ProtectionEvaluation of the Filtration Performance of Cloth Masks and Common Fabric Materials 
Against 20-1000 nm Size Particles. The Annals of Occupational Hygiene54, 789-798, doi:10.1093/annhyg/meq044 (2010).

6 Hill, W. C., Hull, M. S. \& MacCuspie, R. I. Testing of Commercial Masks and Respirators and Cotton Mask Insert Materials using SARS-CoV-2 Virion-Sized Particulates: Comparison of Ideal Aerosol Filtration Efficiency versus Fitted Filtration Efficiency. Nano Letters, doi:10.1021/acs.nanolett.0c03182 (2020).

$7 \quad$ Standard Test Method for Determining the Initial Efficiency of Materials Used in Medical Face Masks to Penetration by Particulates Using Latex Spheres. ASTM International ASTM F2299/F2299M-03 (2017).

8 Determination of Particulate Filter Efficiency Level for N95 Series Filters Against Solid Particulates for Non-Powered, Air-Purifying Respirators Standard Testing Procedure (STP). National Institute for Occupational Safety and Health TEB-APR-STP0059 (2019). 